INPLASY

PROTOCOL

To cite: Wang et al. A systematic review and metaanalysis of the intervention effects of physical exercise on adolescent depression. Inplasy protocol 202170013. doi: 10.37766/inplasy2021.7.0013

Received: 05 July 2021

Published: 05 July 2021

Corresponding author: Xing Wang

wwsus2020@163.com

Author Affiliation:

Shanghai University of Sport

Support: 11DZ2261100T.

Review Stage at time of this submission: Data analysis.

Conflicts of interest:

None declared.

\section{A systematic review and meta-analysis of the intervention effects of physical exercise on adolescent depression}

Wang, X1; Cai, ZD2; Jiang, WT33; Fang, YY4; Sun, WX5; Wang, X6.

Review question / Objective: To systematically evaluate the intervention effect of physical exercise on adolescent depression.

Condition being studied: Retrieved randomized controlled trials (RCTs) on physical exercise intervention in adolescent depression in nine major electronic databases at home and abroad. The search time limit was from the beginning year of inclusion in each database to December 31, 2020. The included literature was evaluated using the PEDro scale. Methodological quality assessment, using Stata16.0 software for Meta analysis, subgroup analysis, sensitivity analysis and publication bias test.

INPLASY registration number: This protocol was registered with the International Platform of Registered Systematic Review and Meta-Analysis Protocols (INPLASY) on 05 July 2021 and was last updated on 05 July 2021 (registration number INPLASY202170013).

\section{INTRODUCTION}

Review question / Objective: To systematically evaluate the intervention effect of physical exercise on adolescent depression.

Condition being studied: Retrieved randomized controlled trials (RCTs) on physical exercise intervention in adolescent depression in nine major electronic databases at home and abroad. The search time limit was from the beginning year of inclusion in each database to December 31,2020 . The included literature was evaluated using the PEDro scale. Methodological quality assessment, using Stata16.0 software for 
Meta analysis, subgroup analysis, sensitivity analysis and publication bias test.

\section{METHODS}

Search strategy: The search strategy adopts a combination of subject terms and free words, and is determined after repeated pre-checks, supplemented by manual retrieval of grey literature, and retrospectively included references when necessary. Chinese search terms include: adolescents, junior high school students, high school students; physical exercise, aerobic exercise, resistance exercise, highintensity interval, physical and mental exercise, yoga, qigong, tai chi, dance, aerobics, running, walking; depression, depression Symptoms, depressive symptoms, depressive emotions, negative emotions; randomized controlled experiments. Taking PubMed as an example for English search terms, the search strategy is as follows: \#1 Adolescent [MeSH Terms] OR Adolescents [Title/Abstract] OR Adolescence [Title/ Abstract] OR Teenager [Title/Abstract] OR Teenagers [Title/Abstract] OR Teen [Title/ Abstract] OR Teens [Title/Abstract] OR Youth [Title/Abstract] OR Youths [Title/ Abstract]; \#2 Exercise [MeSH Terms] OR Physical Exercise [Title/Abstract] OR aerobic exercise [Title/Abstract] OR resistance exercise [Title/Abstract] OR High-intensity interval training [Title/ Abstract] OR mind-body exercise [Title/ Abstract] OR Yoga [Title/Abstract] OR Qi Gong [Title/Abstract] OR Taijiquan [Title/ Abstract] OR Dance [Title/Abstract] OR Running [Title/Abstract] OR Walking [Title/ Abstract]; \#3 Depression [MeSH Terms] OR Depressive Disorder [Title/Abstract] OR Depressive Symptom [Title/Abstract] OR Emotional Depression [Title/Abstract] OR Negative Emotion [Title/Abstract]; \#4 randomized controlled trial [MeSH Terms] \#5 \#1 and \#2 and \#3 and \#4.

Participant or population: Depressed adolescent.

Intervention: Physical exercise.
Comparator: Depressed adolescent.

Study designs to be included: RCT and CR.

Eligibility criteria: According to the PICOS principle of evidence-based medicine, the included individual studies must meet the following eligibility criteria: 1) Research subjects: adolescents aged 12 to 18 who have been diagnosed with depression or have been assessed for depression symptoms through relevant scales. Adolescents with depression: meet the diagnostic and statistical manual of mental disorders (Diagnostic and Statistical Manual of Mental Disorders, DSM-IV/V) or the diagnostic criteria of the International Classification of Diseases (ICD); adolescents with non-clinical depression: not met Diagnosis criteria for depression; the classification of mild to severe depression is based on the classification criteria of each included original experiment. 2) Intervention measures: On the basis of the control group, the experimental group has additional physical exercise intervention; if there are multiple experimental groups in a document, only the physical exercise experimental group is included; if there are multiple physical exercise experimental groups, it is counted as multiple the study. 3) Control measures: The control group has no physical exercise intervention; conventional treatment and activities are in line. 4) Outcome indicators: Use the internationally recognized depressive symptom scale for evaluation, and the score is used as the outcome indicator unit. 5) Research design: The included studies are all Chinese and English randomized controlled trials (RCTs) involving physical exercise intervention in depressed adolescents, and studies using cluster random sampling (C-RCT) are included; all literatures are peer-reviewed Publish original research.

Information sources: The computer system searches nine databases including PubMed, Web of Science, The Cochrane Library, EMbase, Medline, CNKI, China Biomedicine (CBM), VIP and Wan Fang Data, with all retrieval time limits From the 
starting year of inclusion in each database to November 11, 2020.

Main outcome(s): A total of 15 articles and 19 studies with a sample size of 1331 were included. Studies have shown that physical exercise can significantly reduce adolescent depression [SMD $=-0.64,95 \% \mathrm{CI}$ $(-0.89,-0.39), p<0.01]$, with a moderate effect size. Physical exercise has statistical significance in reducing depression in adolescents with depression and nonclinical depression adolescents $(p<0.01$, $p<0.01$ ). Within the subgroups of different depression levels, physical exercise has the best intervention effect on adolescents with mild depression [SMD=-1.19 $(-1.83$, -0.54), $p<0.01$ ], followed by intervention on adolescents with moderate depression [SMD = - $0.97(-1.48,-0.45), p<0.01]$, the intervention effect on adolescents with severe depression level is small [SMD =-0.60 (-0.96, -0.23), p<0.01].

Quality assessment / Risk of bias analysis: Two researchers use the revised version of the PEDro scale to evaluate the methodological quality of the included literature; if there is a conflict, a third party will evaluate it, discuss and reach a consensus. Only if the 10 items of the scale clearly indicate that they meet a certain standard, can they be scored and recorded 1 point; if they do not meet or are not mentioned, the score is 0 ; the total score of the scale is 10 points, " $<4$ " is "quality" "Poor", "4 5" is medium quality, "6 8" is good quality, and "9 10" is high quality.

Subgroup analysis: In order to further explore the source of heterogeneity, a subgroup analysis of potential modifier variables was performed. According to the subgroup analysis of depression category, physical exercise affects depressed adolescents [SMD $=-0.57(-0.90,-0.23)$, $\mathrm{p}<0.01$ ] and non-clinical depressed adolescents [SMD $=-0.67(-1.00,-0.33), p$ $<0.01]$ The intervention effects are statistically significant, indicating that physical exercise can significantly reduce depression in adolescents, and there is a high degree of heterogeneity in the subgroup of non-clinically depressed adolescents. According to the subgroup analysis according to the depression level, physical exercise has mild [SMD $=-1.19$ $(-1.83,-0.54), p<0.01]$, moderate [SMD $=-0.97 \quad(-1.48,-0.45), p<0.01]$ And severe [SMD $=-0.60(-0.96,-0.23), p<0.01]$ depression level adolescent intervention effects are statistically significant, indicating that physical exercise can significantly reduce the depressive symptoms of adolescents with different depression levels, mild depression level adolescents There is a high degree of heterogeneity between the groups. According to the physical exercise form, the subgroup analysis was carried out, aerobic exercise [SMD $=-0.74(-1.03,-0.44)$, $p<0.01]$ and multi-component exercise [SMD=-1.22 (-2.31, -0.14), $p=0.03]$ The intervention effects of yoga [SMD $=-0.12$ $(-0.30,0.70), p=0.22]$ are not obvious, and there is a high degree of heterogeneity among the multi-component exercise groups. According to the exercise cycle, the subgroup analysis was carried out, continuous exercise for 6 to 8 weeks [SMD $=-0.71(-0.98,-0.44), p 12$ weeks [SMD $=-0.76(-1.73,0.21), p=0.12]$ The intervention effect is not obvious, and there is a high degree of heterogeneity between 9-12 weeks and $>12$ weeks. Sex. According to the exercise time for subgroup analysis, $75 \mathrm{~min} /$ time [SMD=-0.73(-1.76, 0.29), $p=0.16$ ] was not obvious, and there was a high degree of heterogeneity among the groups of $>75 \mathrm{~min} /$ time. According to the subgroup analysis of exercise frequency, the intervention effect of 3 4 times/week [SMD $=-0.82(-1.09,-0.54), p<0.01]$ is statistically significant, $<3$ times/week [SMD $=-0.11(-0.29,0.07), p=0.23]$ The intervention effect is not obvious, and there is moderate heterogeneity between groups of 3 to 4 times/week.

Sensitivity analysis: In order to explore whether the heterogeneity between studies is caused by a single study, Stata 16.0 software was used for sensitivity analysis . After eliminating the single studies one by one, the effect size did not change significantly, indicating that the results of the study were relatively stable. 
Country(ies) involved: China.

Keywords: physical exercise; adolescents; depression; meta-analysis; systematic review.

Contributions of each author:

Author 1 - Xiang Wang.

Author 2 - Zhidong Cai.

Author 3 - Wanting Jiang.

Author 4 - Yanyan Fang.

Author 5 - Wenxin Sun.

Author 6 - Xing Wang. 